Meta

Journal des tradlucteurs

Translators' Journal

\title{
De gueules à trois léopards d'or en pal
}

\section{Diane Normand}

Volume 40, numéro 4, décembre 1995

URI : https://id.erudit.org/iderudit/003744ar

DOI : https://doi.org/10.7202/003744ar

Aller au sommaire du numéro

Éditeur(s)

Les Presses de l'Université de Montréal

ISSN

0026-0452 (imprimé)

1492-1421 (numérique)

Découvrir la revue

Citer cet article

Normand, D. (1995). De gueules à trois léopards d'or en pal. Meta, 40(4),

618-623. https://doi.org/10.7202/003744ar d'utilisation que vous pouvez consulter en ligne.

https://apropos.erudit.org/fr/usagers/politique-dutilisation/ 


\section{ÉTUDES TERMINOLOGIQUES ET LINGUISTIQUES}

\section{DE GUEULES A TROIS LEOPARDS D'OR EN PAL}

Est-il à propos, me direz-vous, de traiter un sujet aussi démodé que le sont les armoiries? Bien sûr, ne serait-ce que pour le plaisir. Il est vrai que nous sommes en pleine effervescence technologique, et que technologie et terminologie vont de pair. Je vous propose tout de même un saut terminologique dans l'histoire à la rencontre du blason et de son langage.

L'utilisation des armoiries s'est modifiée au cours des siècles. Elles ont depuis toujours servi à représenter la noblesse et le clergé. Mais au Moyen Âge, plus précisément, elles distinguaient les guerriers au combat. Aujourd hui, elles caractérisent plutôt un lieu géographique (pays, province ou ville). De bouclier à drapeau national, en passant par l'art décoratif. les armoiries ont transporté jusqu'à nos jours une façon de s'exprimer toute particulière, une langue, une syntaxe demeurée inchangée.

\section{D'ARMOIRIES, DE BLASON ET D'HERAI.DIQUE.}

Tout d'abord. yu'en est-il des termes armoiries, blason et héraldique ? Ils ont des traits communs mais comportent des différences à bien connaître.

La science qui étudie les armoiries porte le nom d'héraldique ou de blason. On peut s'y connaître en héraldique et blasonner un écu. c'est-à-dire en expliquer les figures selon les règles de l'art. En anglais, on trouve les expressions heraldry, blazon et hlazomry. Blasonner un écu se dit to blazon a coat.

Armoiries : c'est le symbole dans son ensemble, l'écu accompagné de ses ornements. On l'appelle aussi blason et en anglais coat of arms.

\section{DE DIVISIONS FT DE PIECES HONORABILS}

De quoi se composent les armoiries?

D'un écu (shicld), sorte de bouclier sur lequel sont représentées les figures héraldiques et les pièces honorables (nous y reviendrons). Il peut être de formes diverses : en losange, couché, échancré, ovale ou de type français. C'est ce dernier qui est montré ici. Il s'agit d'un quadrilatère aux angles inférieurs arrondis se rejoignant en pointe au milieu.

Il a deux côtés. appelés la senestre (sinister) et la dextre (dexter) - ou encore. côté senestre el côté dextre - respectivement la gauche et la droite, comme si on portait l'écu sur la poitrine. 


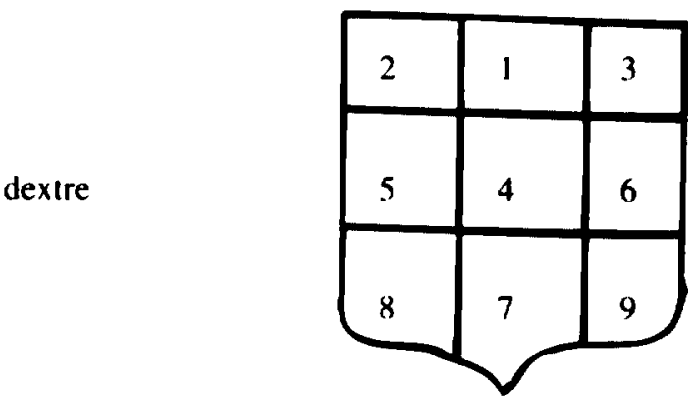

senestre

Représenté ci-dessus, son champ (field), en d'autres mots sa surface, se divise comme suit :

1. Le chef (chief; middle chief):

2. Le canton dextre du chef (dexter chief) :

3. Le canton senestre du chef (sinister chief) :

4. Le cceur ou abìme (fess poimt) ;

5. Le flanc dextre (dexter flank);

6. Le flanc senestre (sinister flank) :

7. La pointe (base) :

8. Le canton dextre de la pointe (dexter base):

9. Le canton senestre de la pointe (sinister hase).

C'est la façon la plus courante de décrire la surface de l'écu. Cependant, le blason peut être divisé en quartiers (quarters) et en cantons (cantons). Le canton, peu fréquent dans les ouvrages anglais consultés, représente le neuvième de la surface, tandis que le quartier correspond au quart.

Viennent ensuite les pièces honorables (ordinaries). Ce sont les formes géométriques de couleurs et d'aspects divers qui divisent l'écu. En voici quelques-unes:

- Le chef (chicf) occupe le tiers horizontal supérieur de l'écu ;

- la fasce (fess; fesse) traverse horizontalement l’écu au milieu :

- le pal (pale) traverse l'écu verticalement au milieu :

- la croix (cross) correspond à la réunion de la fasce et du pal :

— la bande (bend) traverse l'écu diagonalement, de droite à gauche ; et enfín,

- la barre (bend sinister), diagonalement, de gauche à droite.
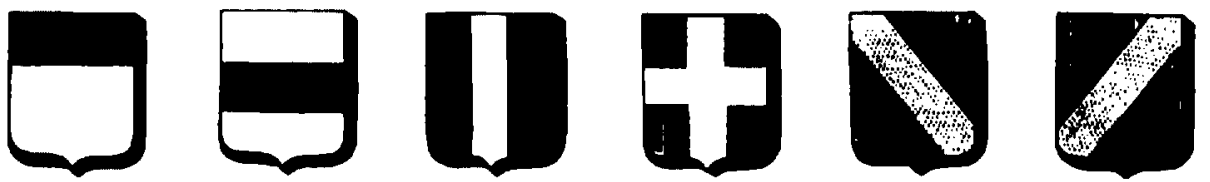

(Nota : Chef a deux sens : l'emplacement sur le champ et la bande de couleur placée à cet endroit sur l'écu.)

Outre les pièces géométriques, l'écu porte des figures ou charges (charges). Celles-ci peuvent être naturelles (humaines, animales, végétales, etc.), artificielles (sceptres, épées, cloches, ancres) ou chimériques (licornes, centaures, dragons). 


\section{D'ORNEMENTS EXTERIEURS}

L'écu peut suffire à représenter les armoiries, mais il est, la plupart du temps, orné. Les pièces qui l'entourent se nomment ornements extérieurs (exterior ornaments).

Il est généralement surmonté d'un heaume (helmêt). nommé aussi casque. ou d'une couronne $((r) w n)$. représentant soit le rang nobiliaire, soit le grade militaire.

Ces couvre-chefs sont parfois entourés de lambrequins (mantlings). qui descendent de part et d'autre de l'écu. Ils ont la forme de lanières, de rubans, de pièces d'étoffe découpées en lambeaux ou encore de cascades de feuilles d'acanthe.

Le torque, tortil ou bourrelet (n'reath) est placé sur le casque. Il s'agit de deux rubans d'étoffe tortillés de la couleur des principaux émaux de l'écu.

Le cimier (crest) est la partic la plus élevée des ornements. Il reproduit parfois une des pièces de l'écu, un animal, par exemple.

L'écu est dit timbré (crested), lorsqu'il est surmonté du heaume ou de la couronne, accompagné ou non du tortil et du cimier.

À dextre et à senestre de l'écu se trouvent des figures qui semblent le tenir ou le supporter. Elles sont appelées tenants lorsqu'il s'agit de figures d'êtres humains, supports lorsqu'il est question de figures animales et soutiens dans le cas d'éléments végétaux. L'anglais ne fait pas cette distinction: dans tous les cas, il emploie le terme supporters.

Le listel ( $\mathrm{s}$ roll : escroll) est un ornement en forme de banderolle ou de ruban. Il est placé sous l'écu et porte la devise (motto). courte sentence faisant allusion au caractère, à la condition ou à un acte de vie de celui qui pone les armoiries.

\section{DE MÉTAUX, DE COUIFURS ET DE FOURRURES}

Les émaux (timcturés) - parce que les armoiries étaient anciennement fabriquées en émail - sont les différentes couleurs dont on revêt le champ de l'écu et les figures qui y sont représentées. En langage héraldique, on distingue deux métaux (métals) et cinq couleurs (colours) proprement dites, portant les noms suivants :

$\begin{array}{cccc} & \text { français } & \text { anglais } & \text { couleur correspondante } \\ \text { MÉTAUX : } & \text { or } & \text { or } & \text { jaune } \\ & \text { argent } & \text { argent } & \text { blanc } \\ \text { COULEURS : } & \text { gueules } & \text { gules } & \text { rouge } \\ & \text { azur } & \text { azure } & \text { bleu } \\ & \text { sinople } & \text { rert } & \text { vert } \\ & \text { pourpre } & \text { purpurc } & \text { violet } \\ & \text { sable } & \text { sable } & \text { noir }\end{array}$

Les fourrures ou pannes (firs) sont en quelque sorte un motif qui recouvre le champ ou une partic de la surface de l'écu. Elles sont au nombre de quatre : l'hermine (ermine), d'argent avec des mouchetures de sable placées en quinconce : la contrehermine (ermines), de sable avec des mouchetures dargent : le vair (vair), clochettes d'azur el d'argent, la base d'une clochette bleue touchant celle d'une clochette blanche : et finalement, le contre-vair (coume'r-1'air), clochettes d'azur et d'argent, la base d'une clochette bleue touchant celle d'une autre clochelte bleue. 

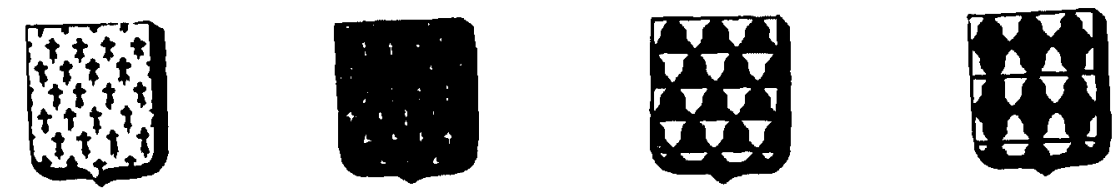

Nous avons tenté de relever quelques faits linguistiques intéressants, de regrouper les termes qui permettent de déchiffrer les armoiries. La nomenclature reste cependant incomplète. C'est pourquoi nous invitons les lecteurs intéressés à consulter les ouvrages cités à la fin de cette courte étude.

\section{LEXIQUE ANGLAIS-FRANCYAIS}

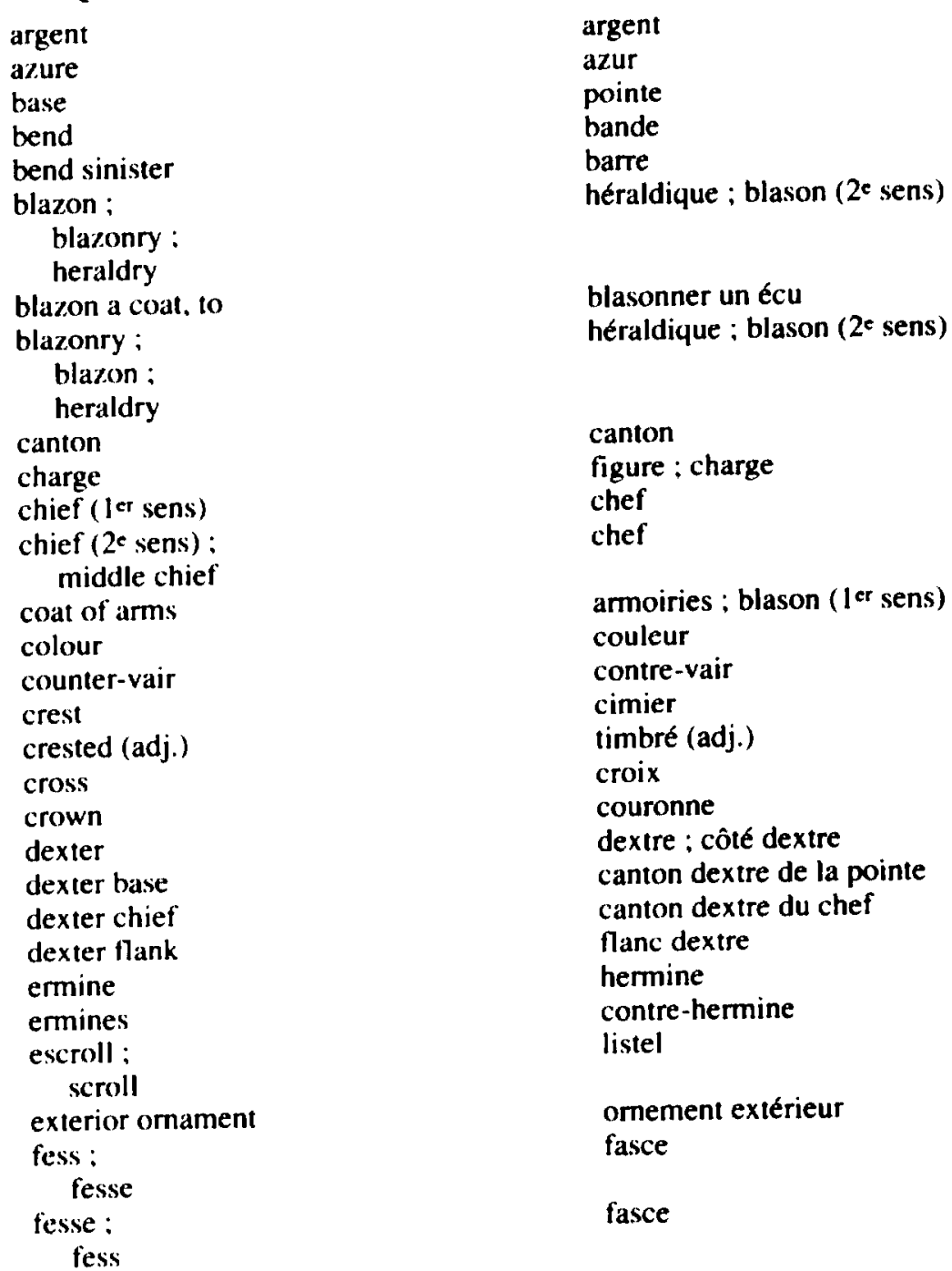




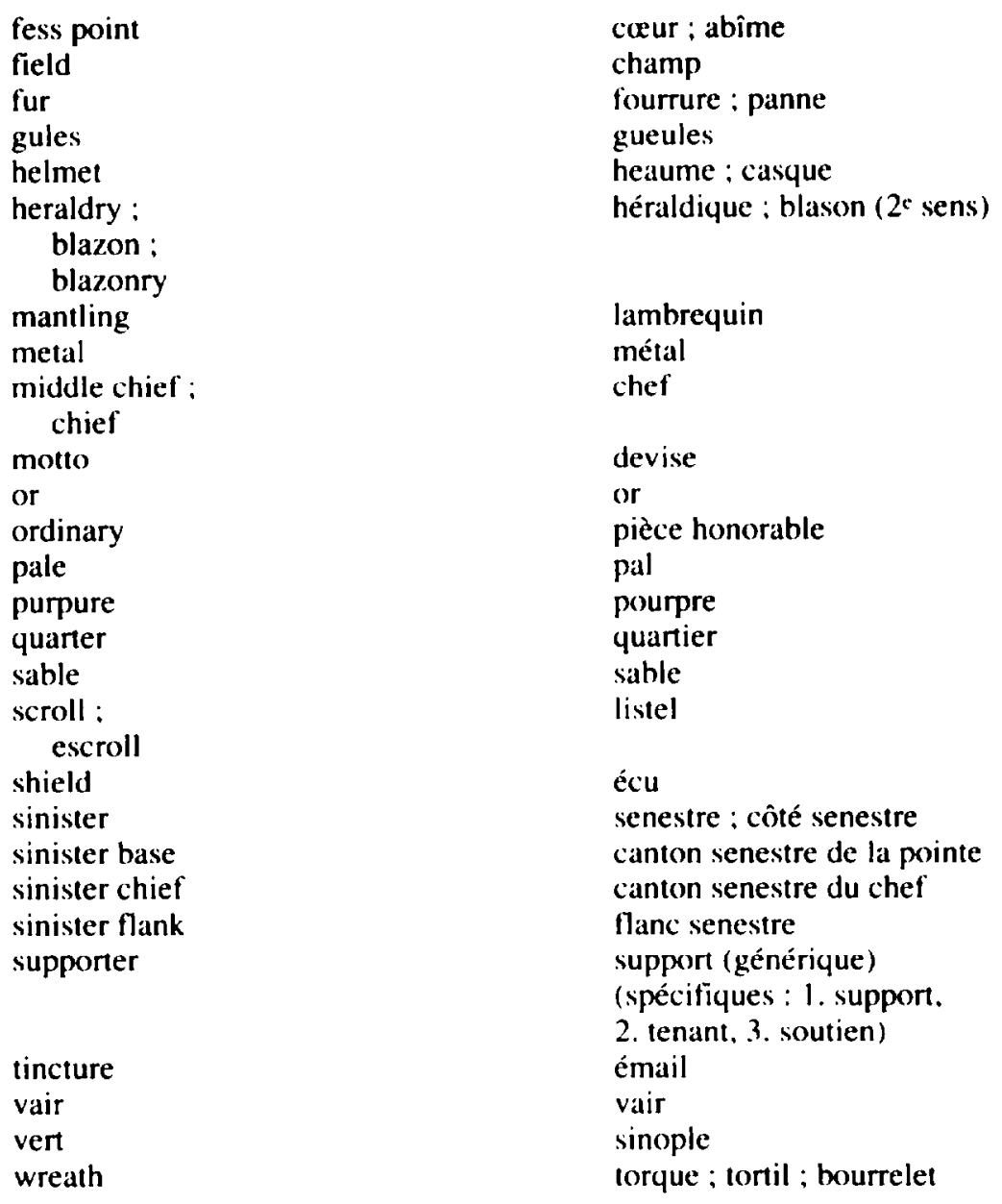

DIANE NORMAND

Momréal. Canada

\section{REFERENCES}

Assemblée nationale (1990) : Less sumbeles d'identité quáhéconise. Les Publications du Québec. ministère des Communications du Quétrec, $39 \mathrm{p}$

BABCOCK COVE, Philip (1968): Webser's Thed New Inernatumal Dictionary, Sprintield. Merriam Company, $2662 \mathrm{p}$.

BRAGONIER. Réginald et David FISHER (198.3) : Le qui est-ce' que dest/Le' what's what la premiere encyclopédic visurelle francos-anglaise. Paris. Éditions RTI., $594 \mathrm{p}$.

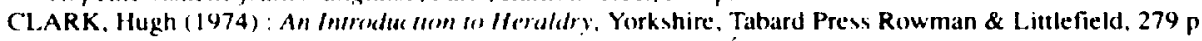

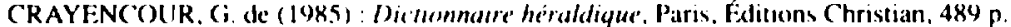

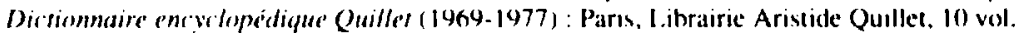

Fincreleperdia Universalis ( $(96 \mathrm{X})$ : Paris, Fencyclopedia (Iniversalis France. $20 \mathrm{vol}$. 
Grand Dicrionnaire Encyclopédique Larousse (1982-1985) : Paris, Librairie Larousse, I0 vol. L.es armoiries, drupeaux et emblèmes du Canada (1981) : Hull, Secrétariat d'État du Canada, 112 p. MAIGNE, W. (1991): Abrégé méthestique de la science des armoiries. Paris, Pardes, 508 p MÉMO LAROUSSE. Encwilupédie générale visuelle ef thémanique (1990) : Paris. Larousse. $1294 \mathrm{p}$ MILBOURNE. J. S. (1908) : Heraldry for amateurs, London, L. Upcoh Gill. 232 p. PASTOURNEAU. Michel ( 1979 ): Traité d' héraldique. Paris. Picard, 368 p.

ROGERS, Col. H. C. B. (1955): The Pageant of Heraldry, London, Seely Service \& Co. Limited, 205 p.

The Now Enc welopedus Britumnica (1980) : U.S.A., Encyclopedia Britannica Inc., 23 vol.

The Random House Dictumury of the English Language (1987): Second Edition. New York, Random House. $2478 \mathrm{p}$.

WIGNIOLE, Jules (1902): Locuhulaire du Blason: supplément de l'Annuaire général héraldique. Paris, Imprimerie Paul Dupont, 112 p. 